\title{
The Stokes boundary layer for a power-law fluid
}

\author{
David Pritchard*, Catriona R. McArdle, Stephen K. Wilson \\ Department of Mathematics and Statistics, University of Strathclyde, 26 Richmond St, \\ Glasgow G1 $1 X H$, Scotland
}

\begin{abstract}
We develop semi-analytical, self-similar solutions for the oscillatory boundary layer ('Stokes layer') in a semi-infinite power-law fluid bounded by an oscillating wall (the so-called Stokes problem). These solutions differ significantly from the classical solution for a Newtonian fluid, both in the non-sinusoidal form of the velocity oscillations and in the manner at which their amplitude decays with distance from the wall. In particular, for shear-thickening fluids the velocity reaches zero at a finite distance from the wall, and for shear-thinning fluids it decays algebraically with distance, in contrast to the exponential decay for a Newtonian fluid. We demonstrate numerically that these semi-analytical, self-similar solutions provide a good approximation to the flow driven by a sinusoidally oscillating wall.
\end{abstract}

Keywords: power-law, Ostwald-de Waele, Stokes's second problem, Stokes layer, oscillatory boundary layer

\section{Introduction}

The Stokes problem, sometimes called Stokes's second problem, is one of the classic boundary-value problems for the unsteady rectilinear flow of a viscous fluid with inertia. In the simplest case, a Newtonian fluid of constant kinematic viscosity $\hat{\nu}$ occupies a semi-infinite space $\hat{y}>0$ bounded by a rigid impermeable wall at $\hat{y}=0$. This wall is oscillated sinusoidally so that the $\hat{x}$ velocity on the wall is given by $\hat{u}(0, \hat{t})=\hat{U} \cos (\hat{\omega} \hat{t})$, while the velocity decays

*Corresponding author. E-mail: david.pritchard@strath.ac.uk. Tel.: $(+44)(0) 141548$ 3819. Fax: $(+44)(0) 1415483345$. 
to zero as $\hat{y} \rightarrow \infty$. The exact solution is given by

$$
\hat{u}(\hat{y}, \hat{t})=\hat{U} \exp (-\hat{\gamma} \hat{y}) \cos (\hat{\omega} \hat{t}-\hat{\gamma} \hat{y}), \quad \text { where } \quad \hat{\gamma}=\sqrt{\frac{\hat{\omega}}{2 \hat{\nu}}}
$$

(see e.g. Batchelor [1], chapter 4; Drazin and Riley [2], §4.1). This structure is sometimes referred to as an oscillatory boundary layer or 'Stokes layer'. It may be visualised as a sinusoidal travelling wave propagating into the fluid at speed $\hat{\omega} / \hat{\gamma}$ and decaying according to an exponential 'envelope' with characteristic thickness $1 / \hat{\gamma}$, though this wavelike behaviour does not imply a dynamical analogy with waves in less dissipative systems.

Apart from its status as an exact solution to the Navier-Stokes equations, the solution (1) provides a useful prototype for more complex flows with oscillatory forcing, such as the boundary layer beneath water waves. An important class of problems in this context concerns the dynamics of the layers of non-Newtonian fluid mud which frequently underlie the clear water in coastal and estuarine settings. These layers may play a key role in wave damping and contribute to the net sediment budget of muddy coasts, but their dynamics are still imperfectly understood (see e.g. McAnally et al. [3] and references therein). An improved understanding of how non-Newtonian fluids behave under oscillatory forcing is therefore of considerable practical interest. In addition to this motivation, Balmforth et al. [4] have recently suggested that the simple but non-viscometric nature of the Stokes boundary layer makes it a useful test for rheological models.

Several previous studies have sought to generalise the classical Stokes problem to non-Newtonian fluids. In particular, analytical and approximate solutions have been presented for several viscoelastic rheologies, including the second- and third-grade fluid models [5, 6], the Johnson-Segalman model [7] and the Burgers model [8]. Although the decay envelopes and wave propagation speeds in these solutions are somewhat modified from the Newtonian case (1), they do not appear to possess qualitatively different features.

Ai and Vafai [9] investigated the Stokes problem numerically for several generalised Newtonian rheologies, including the power-law, Casson and Carreau models. The emphasis of their study was on the transient adjustment from static fluid to a periodic state, and they do not discuss the periodic solutions in any detail. However, there is some suggestion (notably in their figures $3(\mathrm{a})$ and $3(\mathrm{~b})$ ) that in shear-thinning fluids the oscillations die away more gradually with distance from the wall than in shear-thickening fluids. Recently, Balmforth et al. [4] conducted a detailed numerical and experimental 
investigation of the Stokes problem for a viscoplastic Herschel-Bulkley fluid. This problem is particularly intricate because the plasticity means that one or more unyielded 'plug' regions can form.

Perhaps the simplest and most widely used non-linear rheology is the power-law model (see e.g. Barnes et al. [10], §2.3), so it is perhaps surprising that the Stokes problem for such fluids has not been studied in more detail. As we will show below, sinusoidal motion of the wall is not necessarily the most natural boundary condition for this problem, because as the sinusoidal wave propagates away from the wall higher harmonics are generated and it is distorted (as in the viscoplastic flow problem studied by Balmforth et al. [4], in which the velocity of the plug is distinctly non-sinusoidal). However, it is possible to obtain solutions which have the same self-similar structure as the Newtonian solution (1), in that they are periodic at any fixed distance from the wall and that although the amplitude envelope decays away from the wall the oscillatory waveform is unchanged. Numerical evidence suggests that these non-sinusoidal solutions also provide a good approximation to the behaviour of a power-law fluid driven by a sinusoidally oscillating wall. The object of this paper is to demonstrate and expand on these two points.

In $\S 2$ we formulate the mathematical problem and present semi-analytical, self-similar solutions, in which the amplitude envelope and the variation of the phase of the oscillation with $\hat{y}$ are analytically determined while the waveform of the oscillation is obtained as the solution of an ordinary differential equation. In $\S 3$ we compare these solutions with numerical solutions of the governing partial differential equation. Finally, in $\S 4$ we discuss the key features of these solutions and their more general significance.

\section{Semi-analytical solutions}

\subsection{Problem formulation}

In the power-law rheological model (sometimes referred to as the Ostwaldde Waele model), the shear stress $\hat{\tau}$ in a simple shear flow of the kind described in $\S 1$ is given by

$$
\hat{\tau}=\hat{\mu}_{n}\left|\frac{\partial \hat{u}}{\partial \hat{y}}\right|^{n-1} \frac{\partial \hat{u}}{\partial \hat{y}} .
$$

Here $\hat{u}(\hat{y}, \hat{t})$ is the velocity in the $\hat{x}$-direction, parallel to the wall; $\hat{y}$ measures distance from the wall; $\hat{\mu}_{n}$ is a dimensional consistency parameter; and $n>0$ 
is the power-law exponent. The value $n=1$ recovers Newtonian rheology; values of $n<1$ correspond to shear-thinning fluids, such as many colloidal dispersions, in which the effective viscosity decreases with increasing shear; and values of $n>1$ correspond to shear-thickening fluids, such as some polymer solutions, in which the effective viscosity increases with increasing shear. The momentum equation for unsteady rectilinear flow thus becomes a non-linear diffusion equation with the effective viscosity of the fluid acting as a momentum diffusivity,

$$
\hat{\rho} \frac{\partial \hat{u}}{\partial \hat{t}}=\hat{\mu}_{n} \frac{\partial}{\partial \hat{y}}\left(\left|\frac{\partial \hat{u}}{\partial \hat{y}}\right|^{n-1} \frac{\partial \hat{u}}{\partial \hat{y}}\right)
$$

where $\hat{\rho}$ is the fluid density. Throughout, carets will be used to denote dimensional variables and parameters.

It is assumed that the solutions are periodic with angular frequency $\hat{\omega}$, and that some characteristic amplitude $\hat{U}$ may be defined. In the numerical results presented in $\S 3$, the no-slip boundary condition $\hat{u}(0, \hat{t})=\hat{U} \cos (\hat{\omega} \hat{t})$ will be imposed at the oscillating wall, defining $\hat{U}$ directly. In our self-similar solutions we do not prescribe such a boundary condition, but we will assume that an appropriate value of $\hat{U}$ can still be specified. This will be discussed further below.

Finally, we will require that the velocity decays to zero at large distances from the wall, $\hat{u}(\hat{y}, \hat{t}) \rightarrow 0$ as $\hat{y} \rightarrow \infty$.

The problem may now be non-dimensionalised by setting

$$
t=\hat{\omega} \hat{t}, \quad y=\left(\frac{\hat{\omega} \hat{\rho}}{\hat{\mu}_{n} \hat{U}^{n-1}}\right)^{1 /(n+1)} \hat{y} \quad \text { and } \quad u=\frac{\hat{u}}{\hat{U}} .
$$

\subsection{Constructing self-similar solutions}

It is helpful in what follows to note that the governing equation (3) remains unchanged under the transformation $y \mapsto y_{0} \pm y$ for any constant offset $y_{0}$. We will therefore define a new variable $Y=y_{0} \pm y$ and write (3) as

$$
\frac{\partial u}{\partial t}=\frac{\partial}{\partial Y}\left(\left|\frac{\partial u}{\partial Y}\right|^{n-1} \frac{\partial u}{\partial Y}\right) .
$$

If we take $Y=y_{0}+y$ then the far-field boundary condition becomes $u \rightarrow 0$ as $Y \rightarrow \infty$. If we take $Y=y_{0}-y$ then we must either consider solutions that 
are valid for negative values of $Y$ or restrict the domain of the solution to $Y \geq 0$, (i.e. $0 \leq y \leq y_{0}$ ) and impose the condition $u=0$ for all $y \geq y_{0}$. Below we will invariably follow the latter approach, so $Y \geq 0$ implicitly throughout.

Motivated by the Newtonian solution (1), we will seek a solution of the self-similar form

$$
u(Y, t)=Y^{\alpha} f(\eta), \quad \text { where } \quad \eta=t-\phi(Y) \text { for some function } \phi(Y) .
$$

Note that for both definitions of $Y$ we can interpret the offset $y_{0}$ as a boundary-layer thickness. If $Y=y_{0}-y$ then $y_{0}$ represents the thickness of the (finite) layer of non-zero velocity, whereas if $Y=y_{0}+y$ then $y_{0}$ characterises the distance from the wall beyond which the algebraic decay in $y$ asserts itself.

From (6) we immediately obtain

$$
\frac{\partial u}{\partial t}=Y^{\alpha} \frac{\mathrm{d} f}{\mathrm{~d} \eta} \quad \text { and } \quad \frac{\partial u}{\partial Y}=\alpha Y^{\alpha-1} f-Y^{\alpha} \frac{\mathrm{d} \phi}{\mathrm{d} Y} \frac{\mathrm{d} f}{\mathrm{~d} \eta} .
$$

To eliminate $Y$ from the problem, we require that the two terms in $\partial u / \partial Y$ scale in the same way with $Y$. We therefore deduce that

$$
\frac{\mathrm{d} \phi}{\mathrm{d} Y}=\frac{k}{Y} \quad \text { and thus } \quad \phi=k \log (Y),
$$

for some constant $k$ which will in general depend on $n$. (Note that there is no loss of generality in omitting the additive constant of integration, which merely affects the relative phase of the oscillation.) The magnitude of $k$ controls the speed at which the waves propagate, while the sign of $k$ determines the direction in which they propagate. When $k$ is positive, waves travel in the direction of increasing $Y$, and when $k$ is negative, they travel in the direction of decreasing $Y$. Consquently, to ensure that waves travel outwards from the oscillating wall, we will need to take $k>0$ if $Y=y_{0}+y$ and to take $k<0$ if $Y=y_{0}-y$.

Having determined $\phi(Y)$, we find

$$
\frac{\partial u}{\partial Y}=Y^{\alpha-1}\left(\alpha f-k \frac{\mathrm{d} f}{\mathrm{~d} \eta}\right),
$$


so the governing equation (5) becomes

$$
\begin{aligned}
Y^{\alpha} \frac{\mathrm{d} f}{\mathrm{~d} \eta}=n Y^{n(\alpha-1)-1} & \left|\alpha f-k \frac{\mathrm{d} f}{\mathrm{~d} \eta}\right|^{n-1} \\
& \times\left[(\alpha-1)\left(\alpha f-k \frac{\mathrm{d} f}{\mathrm{~d} \eta}\right)-k\left(\alpha \frac{\mathrm{d} f}{\mathrm{~d} \eta}-k \frac{\mathrm{d}^{2} f}{\mathrm{~d} \eta^{2}}\right)\right] .
\end{aligned}
$$

To eliminate $Y$ we now require that

$$
\alpha=n(\alpha-1)-1, \quad \text { i.e. } \quad \alpha=\frac{n+1}{n-1} .
$$

Note that when $n>1$ (a shear-thickening fluid), the exponent $\alpha>0$ : hence to satisfy the far-field condition we must take $Y=y_{0}-y$ and restrict the solution to a layer of finite thickness $0 \leq y \leq y_{0}$. However, when $n<1$ (a shear-thinning fluid), the exponent $\alpha<0$ and we can take $Y=y_{0}+y$ with algebraic decay as $y \rightarrow \infty$.

With this choice of $\alpha$, equation (10) becomes a non-linear ordinary differential equation for the waveform $f(\eta)$,

$$
\frac{\mathrm{d} f}{\mathrm{~d} \eta}=n\left|\alpha f-k \frac{\mathrm{d} f}{\mathrm{~d} \eta}\right|^{n-1}\left[(\alpha-1)\left(\alpha f-k \frac{\mathrm{d} f}{\mathrm{~d} \eta}\right)-k\left(\alpha \frac{\mathrm{d} f}{\mathrm{~d} \eta}-k \frac{\mathrm{d}^{2} f}{\mathrm{~d} \eta^{2}}\right)\right],
$$

which we must solve numerically, subject to the periodicity constraint $f(\eta)=$ $f(\eta+2 \pi)$ for all $\eta$. We can further eliminate the parameter $k$ at the expense of changing the period of the system, by defining

$$
\eta=|k| \eta^{*} \quad \text { and } \quad f=|k|^{-1 /(n-1)} f^{*},
$$

to obtain

$$
\frac{\mathrm{d} f^{*}}{\mathrm{~d} \eta^{*}}=n\left|\alpha f^{*} \mp \frac{\mathrm{d} f^{*}}{\mathrm{~d} \eta^{*}}\right|^{n-1}\left[(\alpha-1)\left(\alpha f^{*} \mp \frac{\mathrm{d} f^{*}}{\mathrm{~d} \eta^{*}}\right) \mp\left(\alpha \frac{\mathrm{d} f^{*}}{\mathrm{~d} \eta^{*}} \mp \frac{\mathrm{d}^{2} f^{*}}{\mathrm{~d} \eta^{* 2}}\right)\right],
$$

where the upper signs correspond to $k>0$ and the lower to $k<0$. If we can find a periodic solution for $f^{*}\left(\eta^{*}\right)$ over $0 \leq \eta^{*} \leq T^{*}$, we can obtain the corresponding periodic solution for $f(\eta)$ over $0 \leq \eta \leq 2 \pi$ by setting $|k|=2 \pi / T^{*}$.

In summary, our self-similar solutions take the form

$$
u(y, t)= \begin{cases}\left(y_{0}-y\right)^{\alpha} f\left(t+|k| \log \left(y_{0}-y\right) ; n\right) & \text { for } \quad n>1, \\ \left(y_{0}+y\right)^{\alpha} f\left(t-|k| \log \left(y_{0}+y\right) ; n\right) & \text { for } \quad n<1,\end{cases}
$$


where the periodic functions $f(\eta ; n)$ and the constants $k(n)$ are obtained numerically and where $\alpha=(n+1) /(n-1)$.

Some additional condition must be specified in order to select the boundary layer thickness $y_{0}$. For example, if the velocity imposed at the wall is precisely of the form $f(t)$ and has amplitude $\hat{U}$, so the oscillation has dimensionless amplitude 1 at $y=0$, then $y_{0}$ must be given by

$$
\left(y_{0} \pm 0\right)^{\alpha} f_{\max }=1 \text {, i.e. } y_{0}=f_{\max }^{-1 / \alpha},
$$

where $f_{\max }=\max _{\eta} f(\eta)$. We will use this choice of $y_{0}$ for convenience when presenting our results in the following section. In general, however, when this self-similar solution acts as an asymptotic approximation to a solution with some different forcing imposed at the wall (as in the numerical integrations we will discuss in $\S 3$ ), a more complicated matching process may be required to determine $y_{0}$. In these circumstances we will take $\hat{U}$ to be specified by the imposed forcing, noting that in this case the self-similar solution will not in general have unit amplitude at the wall.

\subsection{Periodic solutions for shear-thickening fluids $(n>1)$}

We will first consider periodic solutions for shear-thickening fluids $(n>$ 1 ). In this case, the exponent $\alpha<0$ : we must therefore take $Y=y_{0}-y$ to satisfy the far-field condition, and $k<0$ so that waves propagate away from the wall.

Integrating (14) to obtain periodic solutions is slightly complicated by the fact that the equation becomes singular when $\partial u / \partial Y=0$, i.e. along a 'critical line' in phase space on which $\alpha f^{*}+f^{*^{\prime}}=0$. This occurs twice in each period, corresponding to a cusp in the solution for $f^{*}\left(\eta^{*}\right)$. The phase-space trajectories $\left(f^{*}\left(\eta^{*}\right), f^{*^{\prime}}\left(\eta^{*}\right)\right)$ cannot be integrated reliably across the critical line. It is, however, straightforward to show (see Appendix A) that near to a point $\left(f_{0}^{*}, f_{0}^{*^{\prime}}\right)$ on the critical line, a trajectory behaves as

$$
f^{*^{\prime}} \sim f_{0}^{*^{\prime}}+\left(f^{*}-f_{0}^{*}\right)^{1 / n} .
$$

We can then carry out numerical integrations forward in $\eta^{*}$ from a point $\left(-f_{0}^{*}+\epsilon,-f_{0}^{*^{\prime}}+\epsilon^{1 / n}\right)$ and backward in $\eta^{*}$ from a point $\left(f_{0}^{*}+\epsilon, f_{0}^{*^{\prime}}+\epsilon^{1 / n}\right)$, where $0<\epsilon \ll 1$, and vary $f_{0}^{*}$ until the trajectories meet at some point (e.g. $\left.f^{*}=0\right)$. Once these trajectories have been determined above the critical line, the part below the critical line can be completed by symmetry to create a closed trajectory. Figure 1(a) gives examples of the resulting trajectories 
in phase space. If the waveform were sinusoidal the trajectories would be elliptical; in fact they become increasingly non-elliptical as $n$ increases, so $f(\eta)$ adopts an increasingly 'saw-toothed' form (figures $1(\mathrm{~b})$ and $(\mathrm{c})$ ).
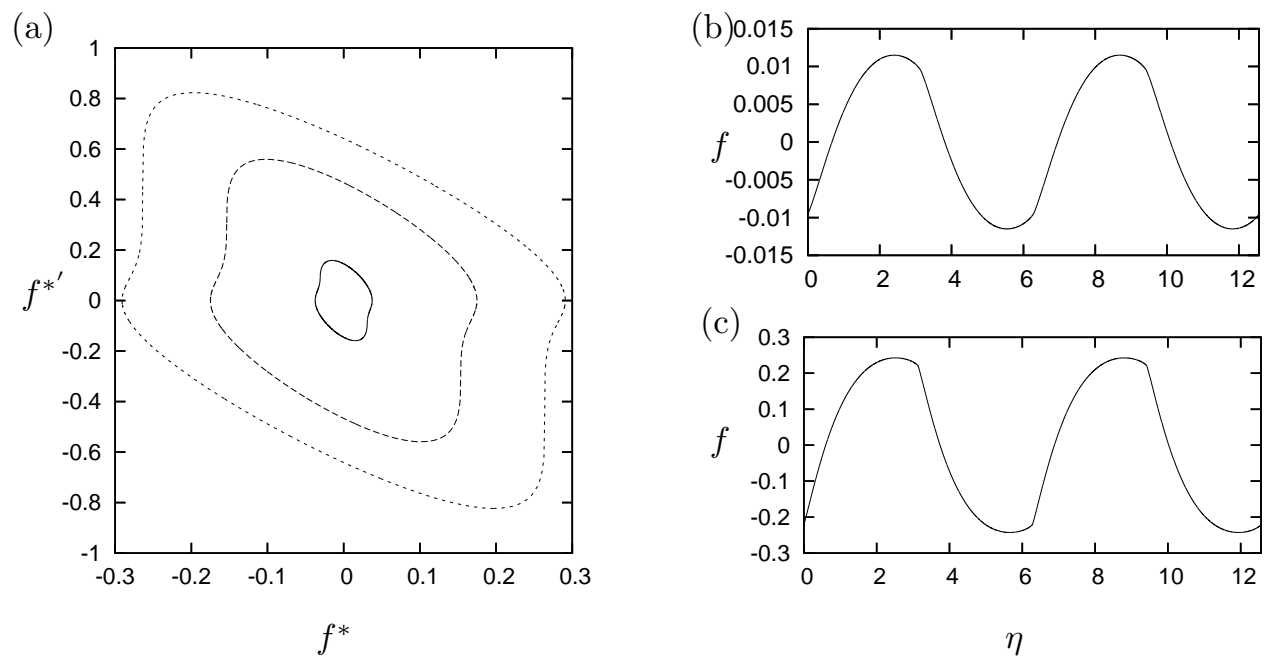

Figure 1: Solutions for $f(\eta)$ for $n=2$ (solid lines), $n=3$ (heavy dashed lines) and $n=4$ (light dashed lines). Figure (a) shows phase-plane portraits $\left(f^{*}, f^{*^{\prime}}\right)$ while figures (b) and (c) show $f(\eta)$ over two periods for the cases (b) $n=2$ and (c) $n=4$.

Having obtained $f^{*}\left(\eta^{*}\right)$, we can determine the period $T^{*}$ from the numerical solutions and use it to deduce the parameter $k$ using $|k|=2 \pi / T^{*}$ and thus, once $y_{0}$ has been determined, the $2 \pi$-periodic solution $f(\eta)$, as in figures $1(\mathrm{~b})$ and (c). Note that by construction, the phase of the oscillations is chosen so that the cusps in $f(\eta)$ occur at $\eta=m \pi$ for $m \in \mathbb{Z}$.

Equipped with $f(\eta)$ and the value of the parameter $k$, we can immediately construct solutions for $u(y, t)$ from (15). These are illustrated, for several values of $n$, in figures $2(\mathrm{~b}-\mathrm{d}$ ) (which show the waveform at various distances from the wall) and in figure 3 (which shows 'snapshots' of the velocity field at various times). In these figures, and elsewhere unless otherwise stated, the value of $y_{0}$ was determined using (16) so the oscillation has amplitude 1 at $y=0$ : this permits direct comparisons to be made between the solutions for different values of $n$.

In figures $2(\mathrm{~b}-\mathrm{d})$ the deviation from a sinusoidal waveform with increasing $n$ is again evident, and comparing these plots with that in the Newtonian 
case (figure 2(a)), the faster decay with $y$ is also evident. As $n$ increases, the phase of the oscillations changes more slowly with $y$ (i.e. the waves propagate more slowly), corresponding to the lower values of $|k|$.

(a)

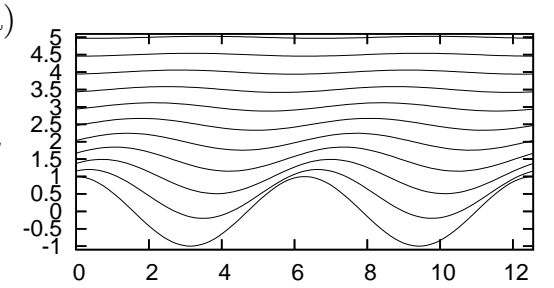

(c)

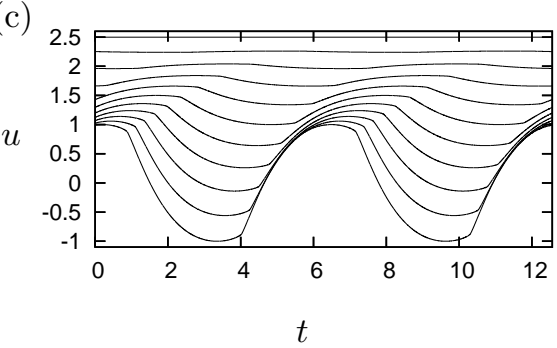

(b)

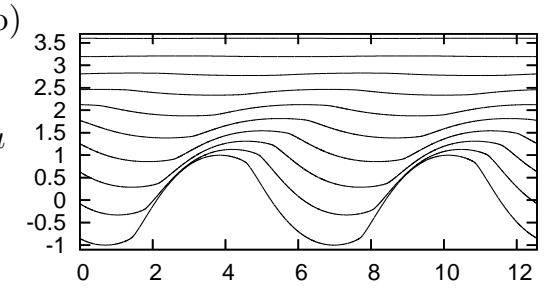

(d)



Figure 2: Solutions for $u(y, t)$ for (a) $n=1$; (b) $n=2\left(k \approx-3.25\right.$; $\left.y_{0} \approx 4.44\right)$; (c) $n=3$ $\left(k \approx-2.13 ; y_{0} \approx 2.89\right)$; and (d) $n=4\left(k \approx-1.72 ; y_{0} \approx 2.34\right)$. Plots are for $y=0$ to $y_{0}$ in 10 equal steps (in (a), for $y=0$ to 5 in 10 equal steps); successive lines in each plot are shifted upwards by equal but arbitrary amounts as $y$ increases.
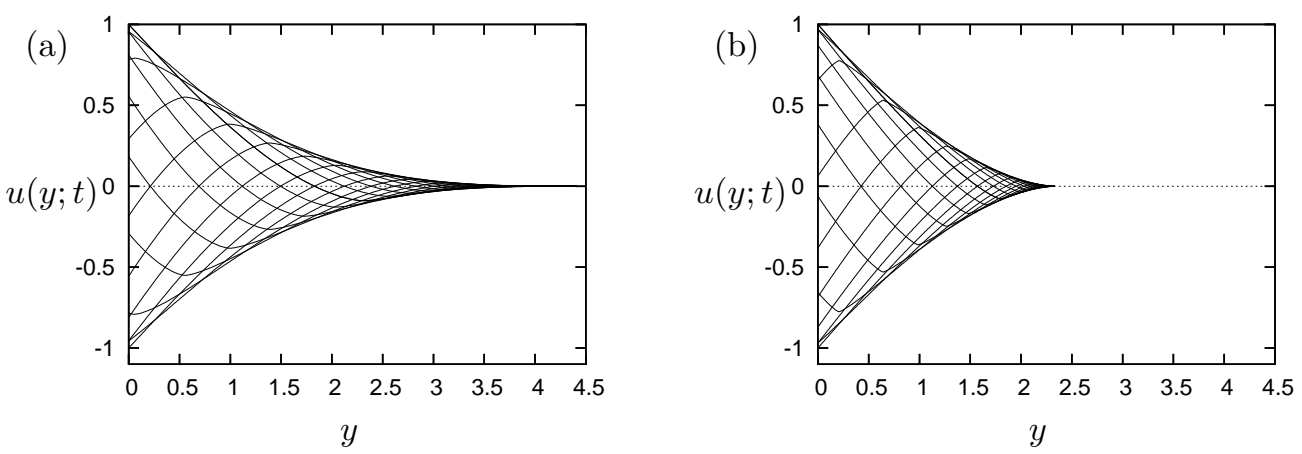

Figure 3: Snapshots of $u(y ; t)$ at time intervals of $\pi / 8$, for (a) $n=2\left(y_{0} \approx 4.44\right)$ and (b) $n=4\left(y_{0} \approx 2.34\right)$. 
The effect of changing $n$ can also be seen in the snapshots in figure 3 . These plots illustrate clearly both the changing envelope, with amplitude decaying more quickly away from $y=0$ for higher $n$, and the increasingly nonsinusoidal waveform, with profiles becoming more angular as $n$ is increased.

\subsection{Periodic solutions for shear-thinning fluids $(n<1)$}

We now consider shear-thinning fluids $(n<1)$, for which the exponent $\alpha<0$. We must therefore take $Y=y+y_{0}$, and $k>0$ so that waves propagate away from the wall. As before, equation (14) is only formally valid away from the critical line where $\partial u / \partial Y=0$. However, the system is not singular on the critical line, in contrast to the shear-thickening problem. This simplifies the numerical integration considerably. However, the closed trajectory which represents periodic solutions appears numerically to be an unstable limit cycle of the ODE, so to obtain it we must integrate backwards in $\eta^{*}$. Apart from this, the construction of the solutions proceeds as before.

(a)

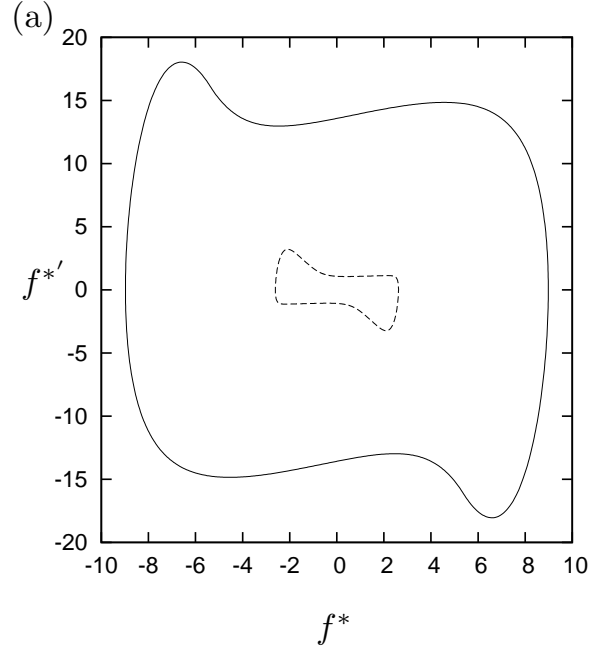

(b)

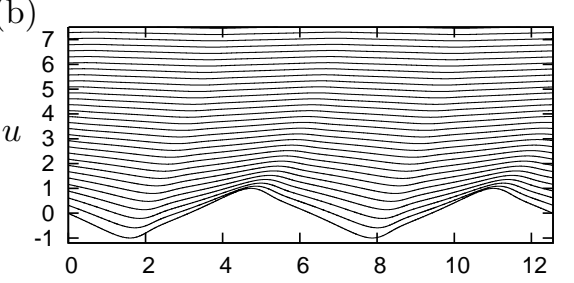

(c)

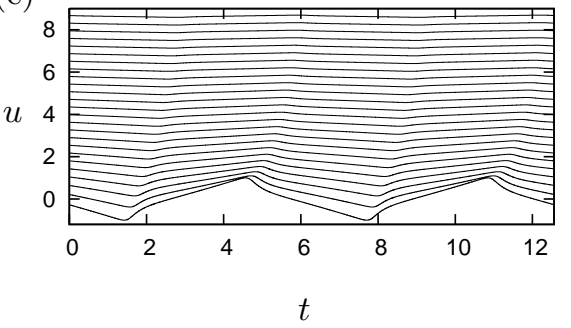

Figure 4: (a) Phase-plane portraits $\left(f^{*}, f^{*^{\prime}}\right)$ for $f(\eta)$ for $n=0.5$ (solid lines) and $n=0.25$ (dashed lines). (b, c) Solutions for $u(y, t)$ for $(\mathrm{b}) n=0.5\left(k \approx 2.242, y_{0} \approx 3.56\right)$; (c) $n=0.25\left(k \approx 0.786, y_{0} \approx 1.47\right)$. Plots are at even increments of $y$ starting at $y=0$; successive lines in each plot are shifted upwards by equal but arbitrary amounts as $y$ increases. 

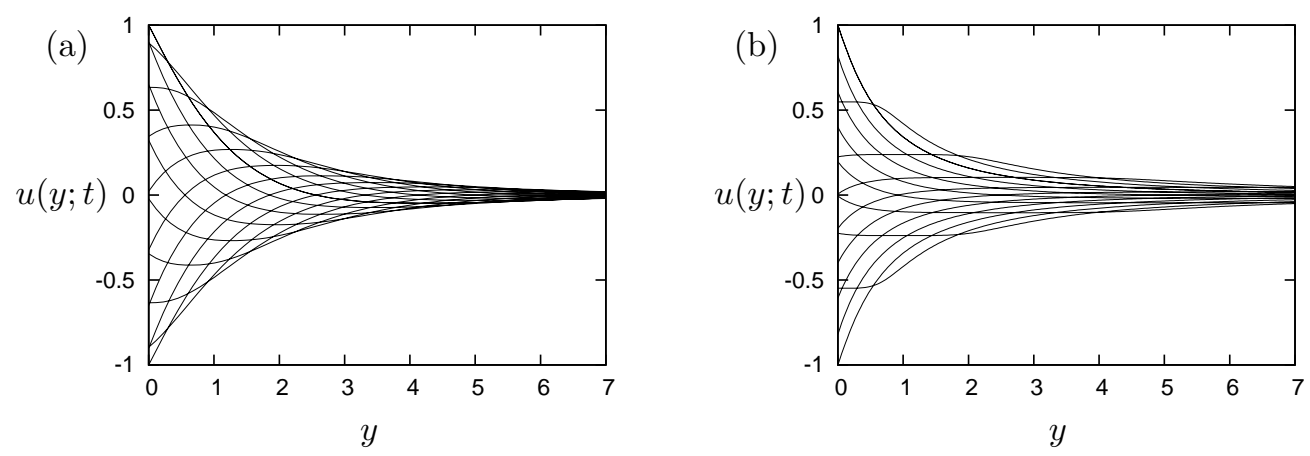

Figure 5: Snapshots of $u(y ; t)$ at time intervals of $\pi / 8$, for (a) $n=0.75\left(y_{0} \approx 9.36\right)$ and (b) $n=0.25\left(y_{0} \approx 1.47\right)$.

Figures 4 and 5 illustrate the results for the two cases $n=0.5$ and $n=$ 0.25. A conspicuous feature is that the waveforms $f(\eta)$ are no longer 'sawtoothed' as they were for shear-thickening fluids (compare figures 4(b) and (c) with figures 1(b) and (c)); rather, they have a nearly triangular 'sharktooth' shape. Conversely, the nature of the snapshot profiles of $u(y ; t)$ (figure 5) has altered: whereas the shear-thickening profiles were highly angular (figure 3), the shear-thinning profiles have more gentle variation and even show extensive regions of near-constant velocity during certain phases (figure $5(\mathrm{~b}))$.

Figure 5 also illustrates the more gentle amplitude decay for shear-thinning than for shear-thickening fluids. This decay becomes noticeable over distances of order $y_{0}$ from the wall: for $y \gtrsim y_{0}$ the decay becomes algebraic with exponent $\alpha<0$. It turns out (see figure 6(b)) that as $n$ decreases, $y_{0}$ becomes smaller (favouring more rapid decay) but $\alpha$ also becomes smaller (favouring less rapid decay); consequently there is not a spectacular visual difference between the decay envelopes for $n=0.25$ and $n=0.75$ shown in figure 5 .

\subsection{Variation of the wavespeed and the boundary layer thickness with $n$}

Figures 6(a) and (b) present more systematically the variation with $n$ of the wavespeed parameter $k$ and of the boundary-layer thickness $y_{0}$ corresponding to unit amplitude at $y=0$. (Figure 6(b) also includes a comparison with the numerical results described in §3.) As the fluid becomes more strongly non-Newtonian, so the value of $n$ differs more from unity, both the 
wavespeed parameter $|k|$ and the boundary layer thickness $y_{0}$ decrease. The limit $n \rightarrow 1$ is singular because in this limit the nondimensional scaling (4) breaks down.
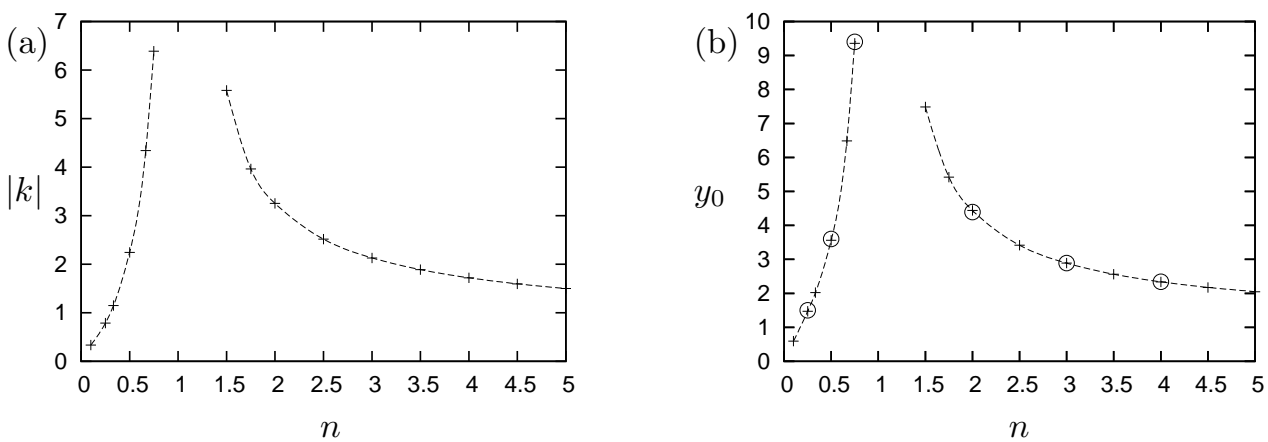

Figure 6: Results for (a) the wavespeed parameter $|k|$ and (b) the boundary layer thickness $y_{0}$ corresponding to unit amplitude at the wall. Dots represent values from the semianalytical solutions; dashed lines are a smoothed fit to these points, and circles in (b) represent the numerical fits $y_{0}^{(\text {num) }}$ reported in table 1 .

\subsection{Shear stress at the wall}

In experiments, it may be simpler to control, or indeed to measure, the shear stress exerted by the wall on the fluid than to measure or control the velocity of the wall directly. It is therefore of interest to consider the shear stress predicted by our semi-analytical solutions.

The dimensionless shear stress at the wall is given by

$$
\tau_{w}(t)=\left.\left|\frac{\partial u}{\partial y}\right|^{n-1} \frac{\partial u}{\partial y}\right|_{y=0}= \pm y_{0}^{2 n /(n-1)}\left|\alpha f(t)-k f^{\prime}(t)\right|^{n-1}\left(\alpha f(t)-k f^{\prime}(t)\right),
$$

where the upper sign corresponds to $Y=y_{0}+y$ (i.e. to shear-thinning cases, $n<1$ ) and the lower sign to $Y=y_{0}-y$ (i.e. to shear-thickening cases, $n>1)$. The corresponding expression for a Newtonian fluid is

$$
\tau_{w}(t)=\left.\frac{\partial u}{\partial y}\right|_{y=0}=\cos \left(t+\frac{5 \pi}{4}\right)
$$

so for a Newtonian fluid the shear stress also varies sinusoidally and its phase leads that of the velocity by $5 \pi / 4$ (see, e.g., Drazin and Riley [2]). 

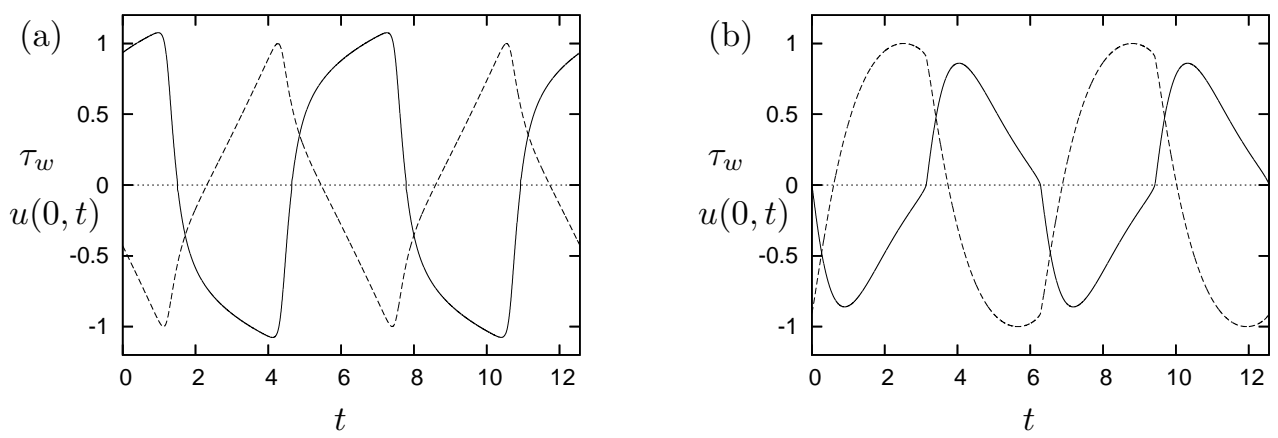

Figure 7: The wall shear stress (solid lines) and wall velocity (dashed lines) for (a) $n=0.25$ and (b) $n=4$.

Figure 7 shows typical wall shear stress histories over two periods for a shear-thinning fluid $(n=0.25)$ and a shear-thickening fluid $(n=4)$. Since the shear stress does not vary with the same waveform as the velocity, a phase difference cannot be defined. However, it is apparent from figure 7 that in both shear-thinning and shear-thickening cases the stress and velocity are roughly in antiphase, with minima of $\tau_{w}$ roughly coinciding with maxima of $u(0, t)$ and vice versa. For shear-thinning fluids (figure $7(\mathrm{a})$ ), a gradual increase in the magnitude of shear stress is followed by a rapid decrease; conversely, for shear-thickening fluids (figure 7(b)), the magnitude of shear stress builds up rapidly and then more gradually diminishes. This qualitative difference may be useful as a means of distinguishing experimentally between shear-thinning and shear-thickening behaviour.

\section{Numerical integration of the full problem}

\subsection{Numerical implementation}

The dimensionless form of the governing equation (5), with $Y \equiv y$, was integrated numerically using the finite-element package Comsol 3.5a [11]. A finite numerical domain $0 \leq y \leq y_{\max }$ was used, with the boundary conditions $u(0, t)=\cos (t)$ and $u\left(y_{\max }, t\right)=0$; the value of $y_{\max }$ was 10 unless otherwise stated. Integrations were started from the initial condition $u(y, 0)=0$ and run for several hundred time units until an effectively periodic state had been attained throughout the domain. The spatial resolution was 121 grid points, and the numerical relative tolerance was $10^{-4}$; the results were robust 
to further refinement of these parameters. Run-times ranged from tens of minutes to several hours on a desktop PC.

The numerical and semi-analytical approaches cannot be expected to yield identical results, both because the numerical method solves an initial value problem rather than seeking perfectly time-periodic solutions, and because the numerical approach imposes a sinusoidal variation at the wall rather than the non-sinusoidal waveforms obtained in $\S 2$. These differences provide a tough test of the semi-analytical solutions, which will be recovered only if they represent the attracting behaviour of the system when it is forced with a 'non-ideal' boundary velocity which is not proportional to the semianalytical waveform $f(\eta)$. If the semi-analytical solutions are attractive then we should expect them to approximate the numerical results best at long times and some distance from the wall.

The two key features predicted by the semi-analytical solutions are the decay envelope of the oscillations and the non-sinusoidal form of the travelling wave. We now consider these features in turn, first for shear-thickening fluids with $n>1$, and then more briefly for shear-thinning fluids with $n<1$.

\subsection{Decay envelope for shear-thickening cases}

Figure 8(a) shows snapshots of the numerical velocity field at large times, for the shear-thickening case $n=4$. The velocity field has become periodic in time almost everywhere, and decays rapidly with distance from the wall. (Note the strong resemblance to figure 3(b), which shows the corresponding semi-analytical results.) This behaviour can be seen more clearly in figure $8(\mathrm{~b})$, where the same data are plotted on a logarithmic scale. In this figure, it is clear that the region of periodic oscillation extends up to $y \approx 2.3$, and beyond this there is a region of small but non-zero velocity which is changing only extremely slowly in time.

The region beyond $y \approx 2.3$ is a relic of the adjustment from the initial condition. Once a finite-thickness boundary layer has formed, the edge of this boundary layer (where the shear rate and thus the effective viscosity vanishes) acts as a barrier to the propagation of further information outward from the wall, and any non-zero flow beyond this point is effectively cut off from the periodic solution. In practice, the numerical method cannot perfectly represent this barrier and a small amount of numerical noise is able to penetrate beyond the boundary layer, but with extremely low amplitudes visible only in figure 8(b). 

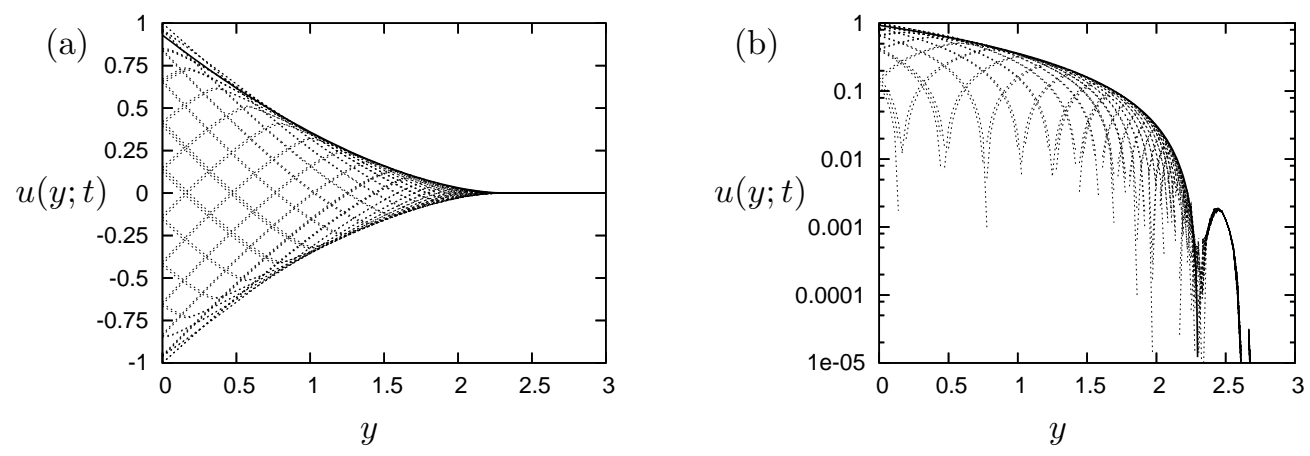

Figure 8: Fitting the envelope to numerical results for $n=4$. Dotted lines are snapshots of $u(y ; t)$ at frequent time intervals; the solid line is the envelope $u_{\max }(y)=a\left(1-y / y_{0}^{(\mathrm{num})}\right)^{\alpha}$ with fitted parameters $a=0.93$ and $y_{0}^{(\text {num })}=2.3$. The two figures show the same data; note the logarithmic scale in (b).

It is easy to fit an envelope of the form $u_{\max }(y)=a\left(1 \mp y / y_{0}^{(\text {num })}\right)^{\alpha}$ to the numerical results. The fitting parameters are tabulated in table 1 , while the fitted values of $y_{0}^{(\text {num })}$ are also marked in figure $6(\mathrm{~b})$. The fitting was carried out by eye: for shear-thickening cases, the region of steep decay in plots such as figure 8(b) unambiguously determines $y_{0}^{(\text {num })}$, and $a$ was then adjusted so that the envelope matched the snapshots as closely as possible. Note that the value of $\alpha$ is determined by the semi-analytical solution and was not used as a fitting parameter; it is included in table 1 for completeness.

\begin{tabular}{|c|c|c|c|c|}
\hline$n$ & $\alpha$ (exact) & $y_{0}($ from $(16))$ & $y_{0}^{(\text {num })}($ fitted $)$ & $a$ (fitted) \\
\hline 0.25 & $-5 / 3$ & 1.47 & 1.5 & 1.15 \\
\hline 0.5 & -3 & 3.56 & 3.6 & 1.10 \\
\hline 0.75 & -7 & 9.36 & 9.4 & 1.02 \\
\hline 2 & 3 & 4.44 & 4.39 & 0.93 \\
\hline 3 & 2 & 2.89 & 2.84 & 0.92 \\
\hline 4 & $5 / 3$ & 2.34 & 2.30 & 0.93 \\
\hline
\end{tabular}

Table 1: Fitted envelope parameters for various shear-thickening and shear-thinning cases. Note that the envelopes were fitted by eye so the fitted parameters may not be optimal.

The numerical boundary layer thickness $y_{0}^{(\mathrm{num})}$ is not necessarily identical to that required for the semi-analytical solution to have unit amplitude at 
the wall, because the numerically imposed boundary condition is sinusoidal rather than having the form $u(0, t) \propto f(t)$. Despite this, the results in table 1 show that in practice $y_{0}^{(\mathrm{num})}$ is rather close to the value of $y_{0}$ given by (16). Similarly, the amplitude $a$ of the fitted envelope at the wall is not necessarily equal to 1 , because the adjustment from sinusoidal to non-sinusoidal waves does not necessarily preserve amplitude. The value of $a$ relative to unity can be used as a crude measure of how much this adjustment influences the solution. The values of $a$ in table 1 are reasonably close to unity and, taken in conjunction with figure 8 , provide convincing evidence that the semianalytical solution provides a good description of the numerically calculated solution despite the issues involved in the adjustment from a sinusoidal to a non-sinusoidal waveform.

\subsection{Waveform for shear-thickening cases}

Figure 9 illustrates the adjustment of the waveform $f(\eta)$ as it propagates away from the wall. At the wall, the imposed numerical waveform is sinusoidal. By $y=0.5$ (figure $9(\mathrm{a})$ ) this has already become distorted, and by $y=1$ (figure 9(b)) the 'saw-tooth' pattern is clear, with gradual increase to a maximum followed by a more rapid decrease. Subsequently the waveform adjusts more gradually towards the semi-analytical prediction (figures $9(\mathrm{c})$ and $(\mathrm{d})$ ), with the cusp becoming more pronounced; by $y=2$ (figure $9(\mathrm{~d})$ ) there is a fairly good match between the numerically calculated and semi-analytical waveforms.

Unfortunately, because the waveform changes as it propagates away from the wall, it is not possible to define unambiguously a local propagation rate and thus to obtain numerical values of $k$ to compare with the semi-analytical results.

\subsection{Decay envelope and waveform for shear-thinning cases}

Similar numerical integrations and fits to the semi-analytical solutions were carried out for a number of shear-thinning cases (see table 1). The adjustment to approximate periodicity was rather more rapid than in the shear-thickening cases: figures 10 and 11 illustrate typical results once periodicity had been attained.

In shear-thinning fluids, because the envelope decays rather slowly with $y$, the boundary condition imposed at $y=y_{\max }$ can have an appreciable effect on the solutions. Indeed, because for low shear stresses the effective 
(a)



(c)

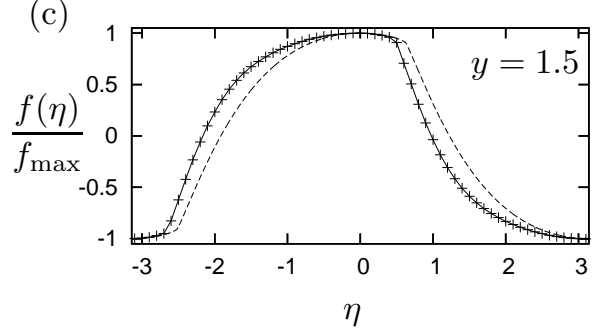

(b)

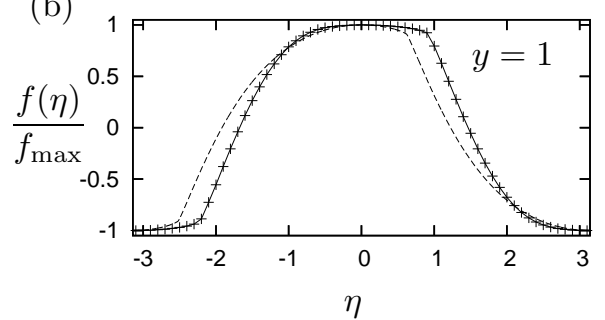

(d)

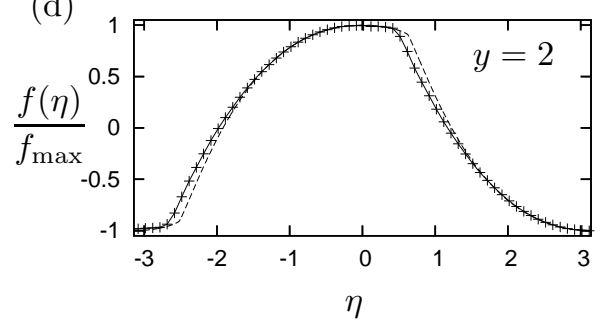

Figure 9: Normalised waveforms $f(\eta) / f_{\max }$ for $n=4$ : crosses are numerical results while dashed lines are the semi-analytical prediction. Numerical data were sampled starting at $t=350$ for values of $y$ as stated in the figures. Phases have been chosen in each case so that the maximum over a period occurs at $\eta=0$.

viscosity (i.e. the momentum diffusivity) is high, the influence of this boundary extends for a considerable distance. The results plotted were obtained for $y_{\max }=25$; comparing these with results for $y_{\max }=10$ (omitted here for brevity) indicated that in the latter case the effect of the boundary was noticeable for $y \gtrsim 6.5$.

The waveforms shown in figure 10 (compare figure 9) illustrate the competing effects of the boundary condition at the wall, the boundary condition at $y=y_{\max }$ and the attracting semi-analytical solution. Close to the wall (figure 10(a)) the imposed sinusoidal waveform can still be felt, although it has already become more angular. As $y$ increases, the waveform adjusts to its semi-analytical 'shark-tooth' form (figure 10(b)), and remains fairly close to this for some distance (figure 10(c)). Eventually, the waveform starts to deviate as the effect of the boundary at $y_{\max }$ is felt, until by $y=20$ (figure $10(\mathrm{~d})$ ) it is significantly different from the semi-analytical prediction.

A similar improvement and then deterioration in agreement between the numerical and semi-analytical solutions can be seen in figure 11, where the semi-analytical envelope is fitted to the snapshot data (compare figure 8). In 

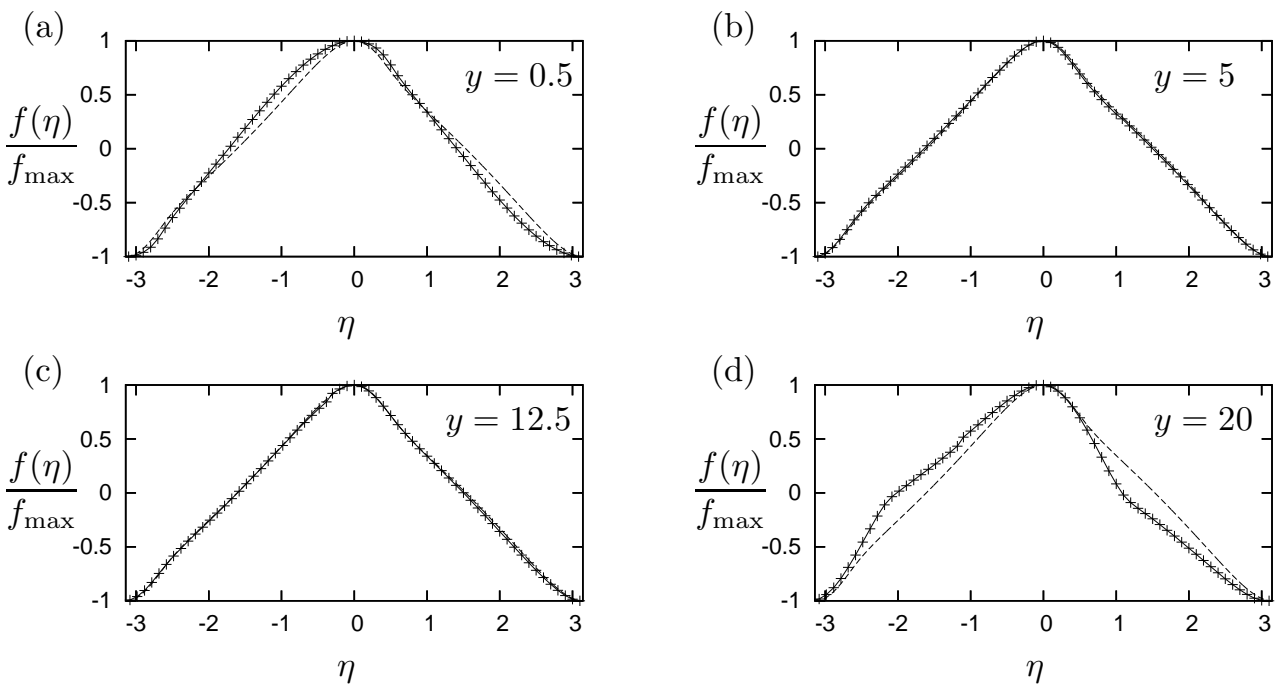

Figure 10: Normalised waveforms $f(\eta) / f_{\max }$ for $n=0.5$, computed on the domain $0 \leq$ $y \leq 25$ : crosses are numerical data while dashed lines are the semi-analytical prediction. Numerical data were sampled starting at $t=30$ for values of $y$ as stated in the figures. Phases have been chosen in each case so that the maximum over a period occurs at $\eta=0$.


Figure 11: Fitting the decay envelope to numerical results for $n=0.5$, computed on the domain $0 \leq y \leq 25$. Dotted lines are snapshots of $u(y ; t)$ at frequent time intervals; the solid line is the envelope $u_{\max }(y)=a\left(1+y / y_{0}^{(\text {num })}\right)^{\alpha}$ with fitted parameters $a=1.10$ and $y_{0}^{(\text {num })}=3.6$. The two figures show the same data set, although more frequent snapshots are shown in (b); also note the logarithmic scale in (b). 
contrast to the shear-thickening case, the fitting here is relatively insensitive to the boundary-layer thickness $y_{0}^{(\text {num })}$, and the region of steep decay is due to the presence of the end wall at $y=25$. There is also some evidence in figure 11(b) of numerical error affecting very low values of $u$. The fit to the analytical envelope is best for the middle part of the domain where the waveforms also agree most closely with the semi-analytical solution, and the amplitude parameter $a$ is now a little greater than unity. These issues notwithstanding, the semi-analytical solution again captures the greater part of the decay reasonably well.

\section{Discussion and conclusions}

We have presented semi-analytical, self-similar solutions for the temporally periodic rectilinear flow of a power-law fluid driven by an oscillating wall. In non-dimensional form, these solutions may be written as

$$
u(y, t)= \begin{cases}\left(y_{0}-y\right)^{\alpha} f\left(t+|k| \log \left(y_{0}-y\right) ; n\right) & \text { for } \quad n>1 \\ \left(y_{0}+y\right)^{\alpha} f\left(t-|k| \log \left(y_{0}+y\right) ; n\right) & \text { for } \quad n<1\end{cases}
$$

where the periodic functions $f(\eta ; n)$ and the constants $k(n)$ are obtained numerically; where $\alpha=(n+1) /(n-1)$; and where $y_{0}$ is determined by matching to the flow driven by the boundary condition at the wall.

The most interesting feature of these solutions is that for shear-thickening fluids $(n>1)$, they predict a boundary layer of finite thickness, with the motion dying out completely beyond a certain distance from the wall. This is reminiscent of the behaviour in the Rayleigh problem for power-law fluids studied by Pascal [12]. It is also reminiscent of the finite thickness of the oscillatory shear layer in the viscoplastic Stokes problem [4]. However, it arises for almost exactly the opposite reason: because the viscosity of a shear-thickening fluid decreases as the shear rate decreases, the viscosity at the edge of the boundary layer is zero and the diffusing signal cannot penetrate further into the fluid. A consequence of this is that in a shearthickening fluid the flow outwith the boundary layer can remain unaffected by the motion of the wall: essentially the fluid has developed an internal slip surface separating non-communicating regions. In a less idealised rheological model with a small but non-zero effective viscosity at zero shear rate, some communication between these regions would be possible. However, the ability of the numerical approach to replicate the slip surface, despite the inevitable 
presence of numerical diffusion, suggests that 'pseudo-slip' behaviour might still be possible.

Conversely, for shear-thinning fluid the influence of the wall extends much further into the fluid than in the Newtonian case, decaying algebraically rather than exponentially with distance from the wall. In the context of fluid mud mobilisation and transport, this behaviour implies that shear-thinning muds may be mobilised to substantially greater depths than a crude estimate based on a Newtonian boundary layer thickness $\hat{\gamma}^{-1}$ would suggest.

Another clear qualitative indicator of shear-thickening or shear-thinning behaviour is the non-sinusoidal waveform of the velocity oscillations. For shear-thickening fluids the oscillations adopt a 'saw-tooth' form, with a rapid decrease in absolute velocity after each minimum or maximum; shear-thinning fluids prefer a 'shark-tooth' waveform which is nearly triangular with rapid reversal at each maximum. The histories of shear stress on the oscillating wall also differ: shear-thinning cases are characterised by gradual increases in stress followed by rapid decreases, whereas shear-thickening cases are characterised by rapid increases in stress followed by more gradual decreases.

These results are not of interest solely as the solutions to a very specific boundary-value problem. Numerical integration of the governing equation with sinusoidal forcing at the wall demonstrates that the self-similar solutions represent attracting states for the system, and so provide a good approximation of the solution even when the forcing is not specifically designed to produce them. For shear-thinning fluids, the influence of the zero-velocity condition at the non-oscillating end wall means that the self-similar solutions are only a good approximation in the middle part of the domain; nevertheless they capture the propagating waveform and the decay envelope reasonably well.

In the present brief study we have necessarily left some avenues unexplored. The transient adjustment to the periodic state is one such; another is the response of the fluid to stress-based, rather than velocity-based, forcing at the wall; a third is the response of fluid layers of finite thickness. There are also obvious opportunities to extend our approach to other rheologies: the Herschel-Bulkley model, which is closely related to the power-law model, is perhaps the most obvious candidate. A final and intriguing possibility, suggested by the experiments of Balmforth et al. [4], is to incorporate thixotropic effects into the rheological model. Work on this even richer problem is currently underway. 
Acknowledgements. We are grateful to two anonymous reviewers for their constructive comments on an earlier version. C. R. McA. is supported by a Doctoral Training Award funded by the Engineering and Physical Sciences Research Council.

\section{Appendix A. Trajectories near the critical line when $n>1$}

For values of $n>1$, the second derivative of $f^{*}$ in equation (14) must become unbounded on the critical line $\alpha f^{*}+f^{*^{\prime}}=0$. (Recall that $k<0$ whenever $n>1$.) We may use the chain rule to write $\mathrm{d}^{2} f^{*} / \mathrm{d} \eta^{* 2}=\mathrm{d} f^{*^{\prime}} / \mathrm{d} \eta^{*}=$ $\left(\mathrm{d} f^{*^{\prime}} / \mathrm{d} f^{*}\right) f^{*^{\prime}}$ so that equation (14) becomes

$$
f^{*^{\prime}}=n\left|\alpha f^{*}+f^{*^{\prime}}\right|^{n-1}\left[(\alpha-1)\left(\alpha f^{*}+f^{*^{\prime}}\right)+\alpha f^{*^{\prime}}+f^{*^{\prime}} \frac{\mathrm{d} f^{*^{\prime}}}{\mathrm{d} f^{*}}\right] .
$$

Setting $f^{*}=f_{0}^{*}+\epsilon$ and taking the ansatz $f^{*^{\prime}}=f_{0}^{*^{\prime}}+\delta_{0} \epsilon^{\beta}$ for some $\beta>0$, where $\alpha f_{0}^{*}+f_{0}^{*^{\prime}}=0$, we obtain

$$
\begin{aligned}
f_{0}^{*^{\prime}}+\delta_{0} \epsilon^{\beta} \sim & n\left|\alpha \epsilon+\delta_{0} \epsilon^{\beta}\right|^{n-1} \\
& \times\left[(\alpha-1)\left(\alpha \epsilon+\delta_{0} \epsilon^{\beta}\right)+\left(f_{0}^{*^{\prime}}+\delta_{0} \epsilon^{\beta}\right)\left(\alpha+\delta_{0} \beta \epsilon^{\beta-1}\right)\right] .
\end{aligned}
$$

Keeping only possible leading-order terms and assuming $f_{0}^{*^{\prime}} \neq 0$, this reduces to

$$
1 \sim n\left|\alpha \epsilon+\delta_{0} \epsilon^{\beta}\right|^{n-1}\left[\alpha+\delta_{0} \beta \epsilon^{\beta-1}\right] .
$$

We now need to consider the size of $\beta$. If $\beta>1$ then the leading-order balance reduces to

$$
1 \sim n|\alpha \epsilon|^{n-1} \alpha,
$$

which is inadmissible because the powers of $\epsilon$ do not balance. On the other hand, if $\beta<1$ then the leading-order balance becomes

$$
1 \sim n\left|\delta_{0} \epsilon^{\beta}\right|^{n-1} \delta_{0} \beta \epsilon^{\beta-1}
$$

which is valid as long as $\beta(n-1)+\beta-1=0$, i.e. $\beta=1 / n<1$. This is consistent; the constant $\delta_{0}=1$ follows immediately, and we have leadingorder behaviour of the form

$$
f^{*}=f_{0}^{*}+\epsilon, \quad f^{*^{\prime}} \sim f_{0}^{*^{\prime}}+\epsilon^{1 / n}
$$

given in (17). 


\section{References}

[1] G. K. Batchelor, An Introduction to Fluid Dynamics, Cambridge University Press, 1967.

[2] P. G. Drazin, N. Riley, The Navier-Stokes Equations: a Classification of Flows and Exact Solutions, number 334 in London Mathematical Society Lecture Notes Series, Cambridge University Press, 2006.

[3] W. H. McAnally, C. Friedrichs, D. Hamilton, E. Hayter, P. Shrestha, H. Rodriguez, A. Sheremet, A. Teeter, Management of fluid mud in estuaries, bays, and lakes. I: Present state of understanding on character and behaviour, Journal of Hydraulic Engineering 133 (2007) 9-22.

[4] N. J. Balmforth, Y. Forterre, O. Pouliquen, The viscoplastic Stokes layer, Journal of Non-Newtonian Fluid Mechanics 158 (2009) 46-53.

[5] K. R. Rajagopal, A note on unsteady unidirectional flows of a nonNewtonian fluid, International Journal of Non-Linear Mechanics 17 (1982) 369-373.

[6] K. R. Rajagopal, T. Y. Na, On Stokes' problem for a non-Newtonian fluid, Acta Mechanica 48 (1983) 233-239.

[7] T. Hayat, S. Asghar, A. M. Siddiqui, Stokes' second problem for a Johnson-Segalman fluid, Applied Mathematics and Computation 148 (2004) 697-706.

[8] M. Khan, A. Anjum, C. Fetecau, On exact solutions of Stokes' second problem for a Burgers fluid, I. The case $\gamma<\lambda^{2} / 4$, Zeitschrift für angewandte Mathematik und Physik 61 (2010) 697-720.

[9] L. Ai, K. Vafai, An investigation of Stokes' second problem for nonNewtonian fluids, Numerical Heat Transfer Part A - Applications 47 (2005) 955-980.

[10] H. A. Barnes, J. F. Hutton, K. Walters, An Introduction to Rheology, Elsevier, 1989.

[11] Comsol, Comsol Multiphysics 3.5a, 2009. See www.comsol.com. 
[12] H. Pascal, Similarity solutions to some unsteady flows of non-Newtonian fluids of power law behaviour, International Journal of Non-Linear Mechanics 27 (1992) 759-771. 